\title{
Erratum
}

\section{Erratum to "Fundamental Solution in the Theory of Thermomicrostretch Elastic Diffusive Solids"}

\section{Rajneesh Kumar and Tarun Kansal}

Department of Mathematics, Kurukshetra University, Kurukshetra 136 119, India

Correspondence should be addressed to Rajneesh Kumar, rajneesh_kukmath@rediffmail.com

Received 20 September 2011; Accepted 11 October 2011

Copyright (C) 2012 R. Kumar and T. Kansal. This is an open access article distributed under the Creative Commons Attribution License, which permits unrestricted use, distribution, and reproduction in any medium, provided the original work is properly cited.

Authors have come across some typographic mistakes in their original paper. Equations (2.1), (2.3), (2.6), (2.9), (3.1), (3.3)-(3.5), (3.7), (3.9), (3.11), (3.13), (3.14), (3.17)-(3.19), (3.22), and (3.35) may be read as

$$
\begin{gathered}
\left(\mu+K^{*}\right) \Delta \overline{\mathbf{u}}+(\lambda+\mu) \operatorname{grad} \operatorname{div} \overline{\mathbf{u}}+K^{*} \operatorname{curl} \overline{\boldsymbol{\varphi}}+x^{*} \operatorname{grad} \overline{\psi^{*}}-\beta_{1} \operatorname{grad} \bar{T}-\beta_{2} \operatorname{grad} \bar{C}=\rho \ddot{\overline{\mathbf{u}}}, \\
\left(f^{*} \Delta-2 K^{*}\right) \overline{\boldsymbol{\varphi}}+\left(\alpha^{*}+\beta^{*}\right) \operatorname{grad} \operatorname{div} \overline{\boldsymbol{\varphi}}+K^{*} \operatorname{curl} \overline{\mathbf{u}}=\rho \dot{\overline{\boldsymbol{\varphi}}}, \\
\left(b^{*} \Delta-c^{*}\right) \overline{\psi^{*}}-x^{*} \operatorname{div} \overline{\mathbf{u}}+g^{*} \bar{T}+h^{*} \bar{C}=\rho \zeta \ddot{\bar{\varphi}}, \\
\left(1+\tau_{0} \frac{\partial}{\partial t}\right)\left(\beta_{1} T_{0} \operatorname{div} \dot{\overline{\mathbf{u}}}+g^{*} T_{0} \dot{\psi^{*}}+\rho C_{E} \dot{\bar{T}}+a T_{0} \dot{\bar{C}}\right)=K \Delta \bar{T}, \\
D \beta_{2} \Delta \operatorname{div} \overline{\mathbf{u}}+D h^{*} \Delta \overline{\psi^{*}}+D a \Delta \bar{T}-D b \Delta \bar{C}+\dot{\bar{C}}+\tau^{0} \ddot{\bar{C}}=0 .
\end{gathered}
$$

$\delta_{1} \Delta \overline{\mathbf{u}}+\delta_{2} \operatorname{grad} \operatorname{div} \overline{\mathbf{u}}+\delta_{3} \operatorname{curl} \overline{\boldsymbol{\varphi}}+\delta_{4} \operatorname{grad} \overline{\psi^{*}}-\operatorname{grad} \bar{T}-\operatorname{grad} \bar{C}=\ddot{\overline{\mathbf{u}}}$,

$$
\begin{gathered}
\left(\delta_{5} \Delta-2 \delta_{3}\right) \overline{\boldsymbol{\varphi}}+\delta_{6} \operatorname{grad} \operatorname{div} \overline{\boldsymbol{\varphi}}+\delta_{3} \operatorname{curl} \overline{\mathbf{u}}=\delta_{7} \ddot{\overline{\boldsymbol{\varphi}}}, \\
\left(\delta_{8} \Delta-\delta_{9}\right) \overline{\psi^{*}}-\delta_{10} \operatorname{div} \overline{\mathbf{u}}+\delta_{11} \bar{T}+\delta_{12} \bar{C}=\ddot{\psi^{*}}, \\
\tau_{t}^{0}\left(\zeta_{2} \operatorname{div} \dot{\overline{\mathbf{u}}}+\zeta_{3} \dot{\overline{\psi^{*}}}+\dot{\bar{T}}+\zeta_{1} \dot{\bar{C}}\right)=\Delta \bar{T}, \\
q_{1}^{*} \Delta \operatorname{div} \overline{\mathbf{u}}+q_{4}^{*} \Delta \overline{\psi^{*}}+q_{2}^{*} \Delta \bar{T}-q_{3}^{*} \Delta \bar{C}+\tau_{c}^{0} \dot{\bar{C}}=0 .
\end{gathered}
$$


$\left(\delta_{1} \Delta+\omega^{2}\right) \mathbf{u}+\delta_{2} \operatorname{grad} \operatorname{div} \mathbf{u}+\delta_{3} \operatorname{curl} \boldsymbol{\varphi}+\delta_{4} \operatorname{grad} \psi^{*}-\operatorname{grad} T-\operatorname{grad} C=\mathbf{0}$,

$\left(\delta_{5} \Delta+\mu^{*}\right) \boldsymbol{\varphi}+\delta_{6} \operatorname{grad} \operatorname{div} \boldsymbol{\varphi}+\delta_{3} \operatorname{curl} \mathbf{u}=\mathbf{0}$,

$-\delta_{10} \operatorname{div} \mathbf{u}+\left(\delta_{8} \Delta+\zeta^{*}\right) \psi^{*}+\delta_{11} T+\delta_{12} C=0$,

$-\tau_{t}^{10}\left[\zeta_{2} \operatorname{div} \mathbf{u}+\zeta_{3} \psi^{*}+\zeta_{1} C\right]+\left(\Delta-\tau_{t}^{10}\right) T=0$,

$q_{1}^{*} \Delta \operatorname{div} \mathbf{u}+q_{4}^{*} \Delta \psi^{*}+q_{2}^{*} \Delta T-q_{3}^{*} \Delta C+\tau_{c}^{10} C=0$.

$$
\begin{gathered}
F_{m n}\left(\mathbf{D}_{\mathbf{x}}\right)=\left[\delta_{1} \Delta+\omega^{2}\right] \delta_{m n}+\delta_{2} \frac{\partial^{2}}{\partial x_{m} \partial x_{n}}, \quad F_{m, n+3}\left(\mathbf{D}_{\mathbf{x}}\right)=F_{m+3, n}\left(\mathbf{D}_{\mathbf{x}}\right)=\delta_{3} \sum_{r=1}^{3} \varepsilon_{m r n} \frac{\partial}{\partial x_{r}}, \\
F_{m 7}\left(\mathbf{D}_{\mathbf{x}}\right)=\delta_{4} \frac{\partial}{\partial x_{m}}, \quad F_{m 8}\left(\mathbf{D}_{\mathbf{x}}\right)=F_{m 9}\left(\mathbf{D}_{\mathbf{x}}\right)=\frac{-\partial}{\partial x_{m}}, \\
F_{m+3, n+3}\left(\mathbf{D}_{\mathbf{x}}\right)=\left(\delta_{5} \Delta+\mu^{*}\right) \delta_{m n}+\delta_{6} \frac{\partial^{2}}{\partial x_{m} \partial x_{n}}, \\
F_{m+3,7}\left(\mathbf{D}_{\mathbf{x}}\right)=F_{7, n+3}\left(\mathbf{D}_{\mathbf{x}}\right)=F_{m+3,8}\left(\mathbf{D}_{\mathbf{x}}\right)=F_{8, n+3}\left(\mathbf{D}_{\mathbf{x}}\right)=F_{m+3,9}\left(\mathbf{D}_{\mathbf{x}}\right)=F_{9, n+3}\left(\mathbf{D}_{\mathbf{x}}\right)=0, \\
F_{7 n}\left(\mathbf{D}_{\mathbf{x}}\right)=-\delta_{10} \frac{\partial}{\partial x_{n}}, \quad F_{77}\left(\mathbf{D}_{\mathbf{x}}\right)=\delta_{8} \Delta+\zeta^{*}, \quad F_{78}\left(\mathbf{D}_{\mathbf{x}}\right)=\delta_{11}, \quad F_{79}\left(\mathbf{D}_{\mathbf{x}}\right)=\delta_{12}, \\
F_{8 n}\left(\mathbf{D}_{\mathbf{x}}\right)=-\zeta_{2} \tau_{t}^{10} \frac{\partial}{\partial x_{n}}, \quad F_{87}\left(\mathbf{D}_{\mathbf{x}}\right)=-\zeta_{3} \tau_{t}^{10}, \quad F_{88}\left(\mathbf{D}_{\mathbf{x}}\right)=\Delta-\tau_{t}^{10}, \\
F_{89}\left(\mathbf{D}_{\mathbf{x}}\right)=-\zeta_{1} \tau_{t}^{10}, \quad F_{9 n}\left(\mathbf{D}_{\mathbf{x}}\right)=q_{1}^{*} \Delta \frac{\partial}{\partial x_{n}}, \quad F_{97}\left(\mathbf{D}_{\mathbf{x}}\right)=q_{4}^{*} \Delta, \quad F_{98}\left(\mathbf{D}_{\mathbf{x}}\right)=q_{2}^{*} \Delta, \\
F_{99}\left(\mathbf{D}_{\mathbf{x}}\right)=-q_{3}^{*} \Delta+\tau_{c}^{10}, \quad m, n=1,2,3 .
\end{gathered}
$$

$\delta_{1} \Delta \mathbf{u}+\delta_{2} \operatorname{grad} \operatorname{div} \mathbf{u}+\delta_{3} \operatorname{curl} \boldsymbol{\varphi}-\delta_{10} \operatorname{grad} \psi^{*}-\zeta_{2} \tau_{t}^{10} \operatorname{grad} T+q_{1}^{*} \Delta \operatorname{grad} C+\omega^{2} \mathbf{u}=\mathbf{H}^{\prime}$.

$$
\begin{gathered}
\delta_{4} \operatorname{div} \mathbf{u}+\left(\delta_{8} \Delta+\zeta^{*}\right) \psi^{*}-\zeta_{3} \tau_{t}^{10} T+q_{4}^{*} \Delta C=Z \\
-\operatorname{div} \mathbf{u}+\delta_{11} \psi^{*}+\left(\Delta-\tau_{t}^{10}\right) T+q_{2}^{*} \Delta C=L \\
-\operatorname{div} \mathbf{u}+\delta_{12} \psi^{*}-\zeta_{1} \tau_{t}^{10} T-q_{3}^{*} \Delta C+\tau_{c}^{10} C=M
\end{gathered}
$$




$$
\begin{gathered}
\left(\Delta+\omega^{2}\right) \operatorname{div} \mathbf{u}-\delta_{10} \Delta \psi^{*}-\zeta_{2} \tau_{t}^{10} \Delta T+q_{1}^{*} \Delta^{2} C=\operatorname{div} \mathbf{H}^{\prime}, \\
\left(v^{*} \Delta+\mu^{*}\right) \operatorname{div} \boldsymbol{\varphi}=\operatorname{div} \mathbf{H}^{\prime \prime} \\
\delta_{4} \operatorname{div} \mathbf{u}+\left(\delta_{8} \Delta+\zeta^{*}\right) \psi^{*}-\zeta_{3} \tau_{t}^{10} T+q_{4}^{*} \Delta C=Z \\
-\operatorname{div} \mathbf{u}+\delta_{11} \psi^{*}+\left(\Delta-\tau_{t}^{10}\right) T+q_{2}^{*} \Delta C=L \\
-\operatorname{div} \mathbf{u}+\delta_{12} \psi^{*}-\zeta_{1} \tau_{t}^{10} T-q_{3}^{*} \Delta C+\tau_{c}^{10} C=M
\end{gathered}
$$

$$
N(\Delta)=\left\|N_{m n}(\Delta)\right\|_{4 \times 4}=\left\|\begin{array}{cccc}
\Delta+\omega^{2} & -\delta_{10} \Delta & -\zeta_{2} \tau_{t}^{10} \Delta & q_{1}^{*} \Delta^{2} \\
\delta_{4} & \delta_{8} \Delta+\zeta^{*} & -\zeta_{3} \tau_{t}^{10} & q_{4}^{*} \Delta \\
-1 & \delta_{11} & \Delta-\tau_{t}^{10} & q_{2}^{*} \Delta \\
-1 & \delta_{12} & -\zeta_{1} \tau_{t}^{10} & -q_{3}^{*} \Delta+\tau_{c}^{10}
\end{array}\right\|_{4 \times 4}
$$

$$
\begin{gathered}
\Psi=\left(\Psi_{1}, \Psi_{2}, \Psi_{3}, \Psi_{4}\right), \quad \Psi_{n}=e^{*} \sum_{m=1}^{4} N_{m n}^{*} d_{m}, \\
\Gamma_{1}(\Delta)=e^{*} \operatorname{det} \mathbf{N}(\Delta), \quad e^{*}=-\frac{1}{q_{3}^{*} \delta_{8}}, n=1,2,3,4 .
\end{gathered}
$$

$$
\left(\Delta+\lambda_{7}^{2}\right) \operatorname{div} \boldsymbol{\varphi}=\frac{1}{v^{*}} \operatorname{div} \mathbf{H}^{\prime \prime} .
$$

$$
\begin{aligned}
\left(\delta_{5} \Delta+\mu^{*}\right)\left[\delta_{1} \Delta \mathbf{u}+\delta_{2} \operatorname{grad} \operatorname{div} \mathbf{u}+\omega^{2} \mathbf{u}\right]+\delta_{3}\left(\delta_{5} \Delta+\mu^{*}\right) \operatorname{curl} \boldsymbol{\varphi} \\
=\left(\delta_{5} \Delta+\mu^{*}\right)\left[\mathbf{H}^{\prime}+\delta_{10} \operatorname{grad} \psi^{*}+\zeta_{2} \tau_{t}^{10} \operatorname{grad} T-q_{1}^{*} \Delta \operatorname{grad} C\right] .
\end{aligned}
$$

$$
\left(\delta_{5} \Delta+\mu^{*}\right)\left[\delta_{1} \Delta \mathbf{u}+\delta_{2} \operatorname{grad} \operatorname{div} \mathbf{u}+\omega^{2} \mathbf{u}\right]+\delta_{3}^{2} \Delta \mathbf{u}-\delta_{3}^{2} \operatorname{grad} \operatorname{div} \mathbf{u}
$$

$$
=\left(\delta_{5} \Delta+\mu^{*}\right)\left[\mathbf{H}^{\prime}+\delta_{10} \operatorname{grad} \psi^{*}+\zeta_{2} \tau_{t}^{10} \operatorname{grad} T-q_{1}^{*} \Delta \operatorname{grad} C\right]-\delta_{3} \operatorname{curl} \mathbf{H}^{\prime \prime} .
$$

$$
\begin{aligned}
\left\{\left[\left(\delta_{5} \Delta\right.\right.\right. & \left.\left.\left.+\mu^{*}\right) \delta_{1}+\delta_{3}^{2}\right] \Delta+\left(\delta_{5} \Delta+\mu^{*}\right) \omega^{2}\right\} \\
= & -\left[\delta_{2}\left(\delta_{5} \Delta+\mu^{*}\right)-\delta_{3}^{2}\right] \operatorname{grad} \operatorname{div} \mathbf{u}+\left(\delta_{5} \Delta+\mu^{*}\right) \\
& \times\left[\mathbf{H}^{\prime}+\delta_{10} \operatorname{grad} \psi^{*}+\zeta_{2} \tau_{t}^{10} \operatorname{grad} T-q_{1}^{*} \Delta \operatorname{grad} C\right]-\delta_{3} \operatorname{curl} \mathbf{H}^{\prime \prime} .
\end{aligned}
$$




$$
\begin{aligned}
\Gamma_{1}(\Delta)\left[\delta_{5} \delta_{1} \Delta^{2}+\left(\mu^{*} \delta_{1}+\delta_{5} \omega^{2}+\delta_{3}^{2}\right) \Delta+\mu^{*} \omega^{2}\right] \mathbf{u} \\
=-\left[\delta_{2}\left(\delta_{5} \Delta+\mu^{*}\right)-\delta_{3}^{2}\right] \operatorname{grad} \Psi_{1}+\left(\delta_{5} \Delta+\mu^{*}\right) \\
\quad \times\left[\Gamma_{1}(\Delta) \mathbf{H}^{\prime}+\delta_{10} \operatorname{grad} \Psi_{2}+\zeta_{2} \tau_{t}^{10} \operatorname{grad} \Psi_{3}-q_{1}^{*} \Delta \operatorname{grad} \Psi_{4}\right]-\delta_{3} \Gamma_{1}(\Delta) \operatorname{curl} \mathbf{H}^{\prime \prime}
\end{aligned}
$$

$$
\begin{aligned}
\Psi^{\prime}=f^{*}\{-[ & \left.\delta_{2}\left(\delta_{5} \Delta+\mu^{*}\right)-\delta_{3}^{2}\right] \operatorname{grad} \Psi_{1}+\left(\delta_{5} \Delta+\mu^{*}\right) \\
& \left.\times\left[\Gamma_{1}(\Delta) \mathbf{H}^{\prime}+\delta_{10} \operatorname{grad} \Psi_{2}+\zeta_{2} \tau_{t}^{10} \operatorname{grad} \Psi_{3}-q_{1}^{*} \Delta \operatorname{grad} \Psi_{4}\right]-\delta_{3} \Gamma_{1}(\Delta) \operatorname{curl} \mathbf{H}^{\prime \prime}\right\}
\end{aligned}
$$

$$
\begin{aligned}
& q_{11}(\Delta)=f^{*} e^{*}\left\{\left(\delta_{5} \Delta+\mu^{*}\right)\left[\delta_{10} N_{12}^{*}+\zeta_{2} \tau_{t}^{10} N_{13}^{*}-q_{1}^{*} \Delta N_{14}^{*}\right]-\left(\delta_{2}\left(\delta_{5} \Delta+\mu^{*}\right)-\delta_{3}^{2}\right) N_{11}^{*}\right\}, \\
& q_{21}(\Delta)=-f^{*} \delta_{3} \Gamma_{1}(\Delta), \quad q_{12}(\Delta)=-f^{*} \delta_{3}\left(\Delta+\lambda_{7}^{2}\right), \quad q_{22}(\Delta)=-\frac{f^{*}}{v^{*}}\left[\delta_{6}\left(\delta_{1} \Delta+\omega^{2}\right)-\delta_{3}^{2}\right], \\
& q_{31}(\Delta)=f^{*} e^{*}\left\{\left(\delta_{5} \Delta+\mu^{*}\right)\left[\delta_{10} N_{22}^{*}+\zeta_{2} \tau_{t}^{10} N_{23}^{*}-q_{1}^{*} \Delta N_{24}^{*}\right]-\left(\delta_{2}\left(\delta_{5} \Delta+\mu^{*}\right)-\delta_{3}^{2}\right) N_{21}^{*}\right\}, \\
& q_{41}(\Delta)=f^{*} e^{*}\left\{\left(\delta_{5} \Delta+\mu^{*}\right)\left[\delta_{10} N_{32}^{*}+\zeta_{2} \tau_{t}^{10} N_{33}^{*}-q_{1}^{*} \Delta N_{34}^{*}\right]-\left(\delta_{2}\left(\delta_{5} \Delta+\mu^{*}\right)-\delta_{3}^{2}\right) N_{31}^{*}\right\}, \\
& q_{51}(\Delta)=f^{*} e^{*}\left\{\left(\delta_{5} \Delta+\mu^{*}\right)\left[\delta_{10} N_{42}^{*}+\zeta_{2} \tau_{t}^{10} N_{43}^{*}-q_{1}^{*} \Delta N_{44}^{*}\right]-\left(\delta_{2}\left(\delta_{5} \Delta+\mu^{*}\right)-\delta_{3}^{2}\right) N_{41}^{*}\right\}, \\
& q_{13}(\Delta)=e^{*} N_{12}^{*}, \quad q_{14}(\Delta)=e^{*} N_{13}^{*}, \quad q_{15}(\Delta)=e^{*} N_{14}^{*}, \\
& q_{33}(\Delta)=e^{*} N_{22}^{*}, \quad q_{34}(\Delta)=e^{*} N_{23}^{*}, \quad q_{35}(\Delta)=e^{*} N_{24}^{*}, \\
& q_{43}(\Delta)=e^{*} N_{32}^{*}, \quad q_{44}(\Delta)=e^{*} N_{33}^{*}, \quad q_{45}(\Delta)=e^{*} N_{34}^{*}, \\
& q_{53}(\Delta)=e^{*} N_{42}^{*}, \quad q_{54}(\Delta)=e^{*} N_{43}^{*}, \quad q_{55}(\Delta)=e^{*} N_{44}^{*} .
\end{aligned}
$$

The above errors do not affect the analytic results of the paper. Inconvenience caused is regretted. 


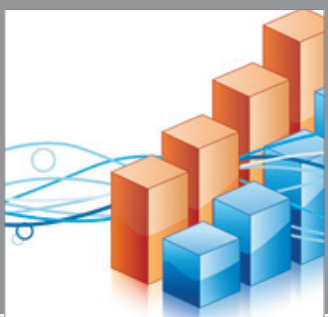

Advances in

Operations Research

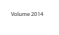

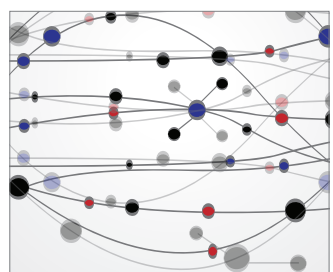

\section{The Scientific} World Journal
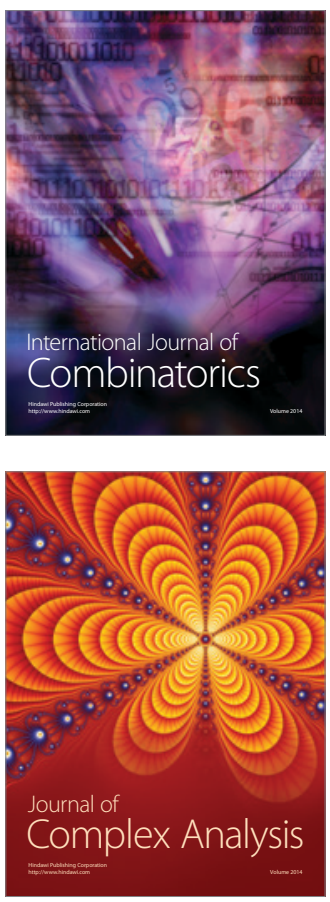

International Journal of

Mathematics and

Mathematical

Sciences
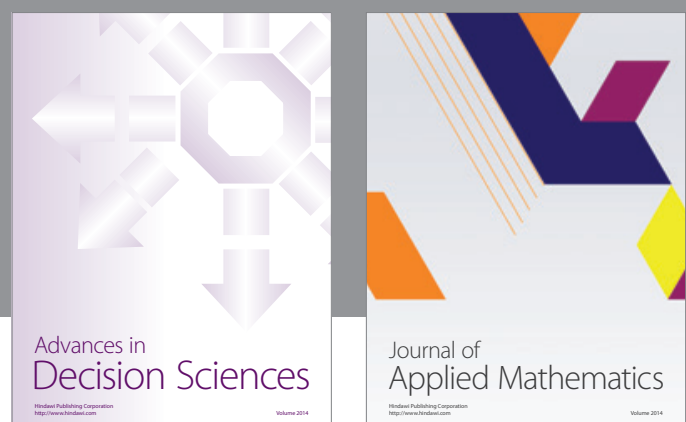

Journal of

Applied Mathematics
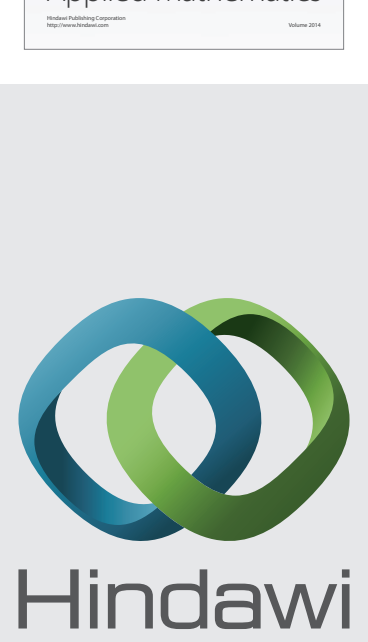

Submit your manuscripts at http://www.hindawi.com
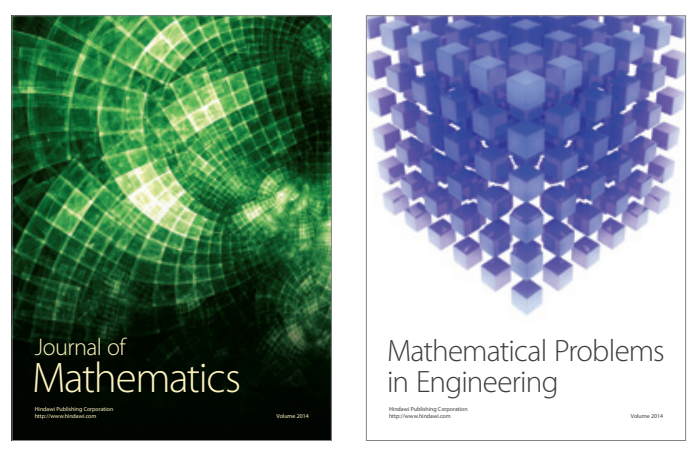

Mathematical Problems in Engineering
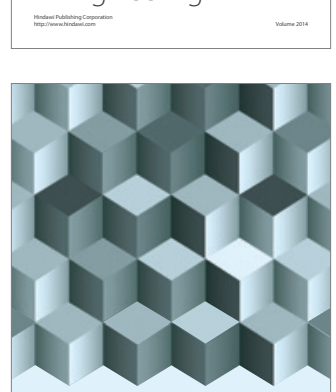

Journal of

Function Spaces
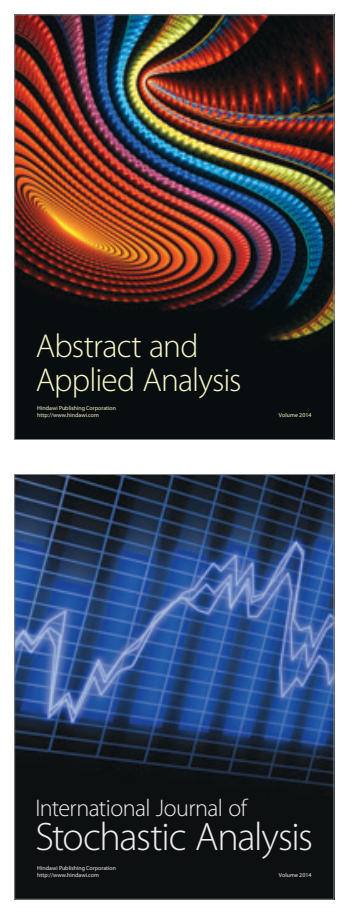

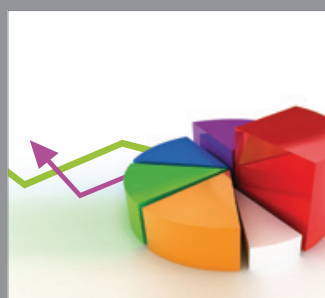

ournal of

Probability and Statistics

Promensencen
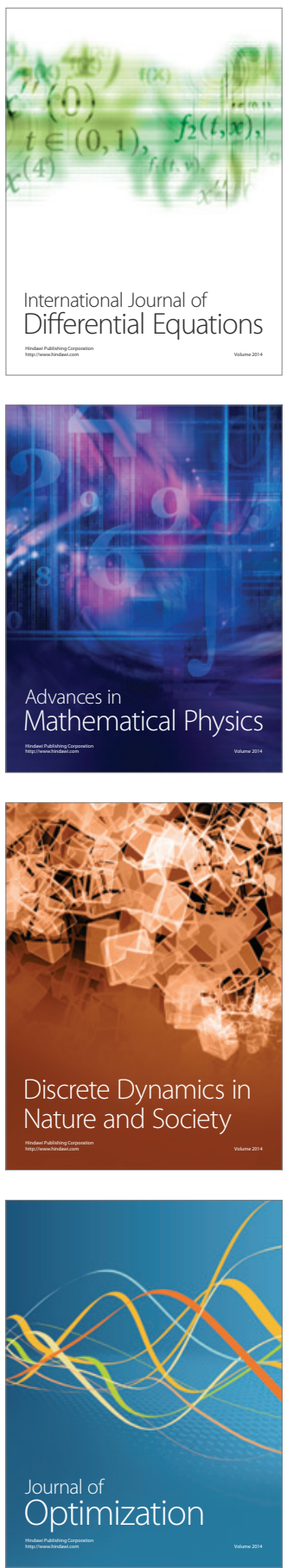